\title{
Sur la distribution de l'article défini dans les constructions superlatives en bulgare
}

On the Distribution of the Definite Article in Superlative Constructions in Bulgarian

\section{Vassil Mostrov}

\section{(2) OpenEdition}

Journals

Édition électronique

URL : https://journals.openedition.org/scolia/1734

DOI : $10.4000 /$ scolia. 1734

ISSN : 2677-4224

Éditeur

Presses universitaires de Strasbourg

Édition imprimée

Date de publication : 9 juillet 2021

Pagination : 93-124

ISBN : 979-10-344-0091-1

ISSN : 1253-9708

\section{Référence électronique}

Vassil Mostrov, «Sur la distribution de l'article défini dans les constructions superlatives en bulgare», Scolia [En ligne], 35 | 2021, mis en ligne le 09 juillet 2021, consulté le 14 juillet 2021. URL : http:// journals.openedition.org/scolia/1734; DOI : https://doi.org/10.4000/scolia.1734

\section{(c) (i) 8 (9)}

Les contenus de la revue Scolia sont mis à disposition selon les termes de la Licence Creative Commons Attribution - Pas d'Utilisation Commerciale - Partage dans les Mêmes Conditions 4.0 International. 
SCOLIA 35/2021, p. 93-124.

\title{
Sur la distribution de l'article défini dans les constructions superlatives en bulgare
}

\author{
Vassil Mostrov* \\ Université Polytechnique Hauts-de-France \\ UR DeScripto \\ vassil.mostrov@uphf.fr
}

В памет на най-добрия башза «À la mémoire du meilleur des pères»

\section{Introduction}

Ce travail est consacré à l'expression du superlatif en bulgare et aux conditions dans lesquelles il peut être accompagné ou non de l'article défini. Après un bref aperçu de la littérature sur le sujet (cf. 1.), nous présenterons de façon générale la catégorie du superlatif dans cette langue en la différenciant du comparatif (cf. 2.). Nous nous focaliserons ensuite (cf. 3.) sur l'analyse des cas où le mot gradable est un adjectif qualificatif, en distinguant les emplois épithétiques des emplois attributifs, ce qui nous permettra de faire un pas en avant vers la compréhension de la distribution de l'article défini. En effet, nous verrons que celui-ci ne peut apparaitre en bulgare qu'en la présence d'une tête nominale (vide ou réalisée). Seront examinés différents contextes (qui exigent, proscrivent ou encore rendent facultatif l'usage du défini) et la lecture du superlatif qui leur est associée: absolue, relative (Szabolcsi, 1986; Heim, 1999) ou encore élative. Nous montrerons que la présence de l'article défini dépend en

* Je remercie chaleureusement Marleen Van Peteghem de m'avoir fait découvrir ce sujet et de m'avoir apporté son aide précieuse sans laquelle ce travail - loin d'être parfait - n'aurait jamais vu le jour. 
grande partie de la portée du morphème superlatif (à l'intérieur $v s$. à l'extérieur du syntagme superlatif).

Même si cette recherche porte sur le bulgare, nous exploiterons des données d'autres langues, notamment le français, l'italien, l'espagnol, le roumain et l'anglais, puisque de nombreux travaux y sont consacrés (Loccioni, 2018 pour l'italien; Coppock \& Strand, 2017; Matushansky, 2008 pour d'autres langues), ce qui n'est pas le cas du bulgare. Les données et les analyses montreront qu'en définitive le bulgare appartient aux langues où l'article défini n'est pas structurellement associé au morphème du superlatif, mais qu'il est utilisé (pour exprimer l'unicité et la présupposition, cf. Kleiber, 1992) quand la structure syntaxique le permet ou l'exige.

Nous empruntons, dans nos analyses, certains concepts et mécanismes syntaxiques à la grammaire générative, cadre théorique privilégié dans les travaux dont nous nous sommes inspiré (d'où l'utilisation d'étiquettes comme DP, NP ou DegP'). Toutefois, quand le recours à la théorie générativiste n'est pas indispensable, nous adoptons des approches plus "classiques», comme celles de la grammaire traditionnelle et de la sémantique lexicale.

\section{Bref aperçu de la littérature}

En Bulgarie, il y a deux chercheurs qui se sont penchés sur la question de l'expression des degrés: Burov, qui y a consacré sa thèse de doctorat (1981) et qui a également publié plusieurs travaux sur le sujet (dont Burov, 1977, 1982) et Choroleeva, qui est l'auteure d'une monographie sur la catégorie des degrés dans une perspective sémantique et lexicologique (2007). Même si ces travaux sont très riches et donnent un aperçu global de la question, le lien entre le superlatif et la définitude n'y est pas véritablement abordé: dans

1 DP (Determiner Phrase, Syntagme Déterminatif) (Abney, 1987) est une étiquette utilisée pour les "syntagmes nominaux» dont le déterminant (D) - qui en est la tête - permet l'actualisation, dans le discours, du nom ou du complexe nom + modifieur. En revanche, NP (Nominal Phrase) correspond à la séquence nominale non actualisée, dépourvue de déterminant. DegP (Degree Phrase) (Krasikova, 2012) est une projection dont la tête est un déterminant défini (exprimé ou non), lequel marque l'unicité du degré exprimé par le superlatif, sans (forcément) donner lieu à un DP. 
ses différents travaux, Burov remarque que le superlatif est le plus souvent accompagné de l'article défini et, même s'il donne des exemples de SN superlatifs non définis, ils ne sont pas clairement distingués de ceux qui ont une interprétation élative. De son côté, Choroleeva (2007: 161) précise que l'unicité est associée à l'expression du superlatif, mais sans faire de lien avec l'usage de l'article défini. Elle fait par contre une remarque intéressante concernant les superlatifs absolus (au sens traditionnel du terme), à savoir qu'ils ne dépendent pas en eux-mêmes de la catégorie de la définitude/indéfinitude. Autrement dit, les syntagmes où ils apparaissent peuvent être définis ou indéfinis en fonction de leur position syntaxique dans la phrase ou de l'interprétation de celle-ci (spécifique, existentielle ou autre). Bien que la situation des «vrais» superlatifs soit plus complexe, nous verrons qu'ils suivent grosso modo la même tendance.

Quant aux différentes grammaires consultées (nous n'en retenons que deux: Tilkov et al., 1983; Pašov, 2015), elles se limitent principalement à la différenciation de la formation du comparatif et du superlatif. Pour ce qui est de l'article défini, elles signalent simplement que le superlatif en est souvent accompagné, en donnant des exemples décontextualisés, et sans expliciter les conditions de l'alternance absence/présence de défini, ni les différences interprétatives qui en découlent.

Pour ce qui est des travaux publiés ailleurs qu'en Bulgarie, signalons l'article (dans le domaine de la sémantique formelle) de Pancheva \& Tomaszewicz (2012), qui compare le bulgare, le polonais et l'anglais au niveau de la disponibilité de différentes lectures relatives en fonction de la présence ou de l'absence de l'article défini et de la portée de celui-ci. C'est d'ailleurs le seul travail sur le bulgare où la question du lien entre l'article défini et le superlatif soit abordée, mais son objectif étant très précis, peu de cas sont finalement étudiés.

\section{Le superlatif en bulgare: généralités}

\subsection{Différencier le superlatif du comparatif}

Comme l'anglais et le latin, le bulgare appartient aux langues qui ont des morphèmes distincts pour le comparatif et le superlatif. On 
utilise, pour le comparatif, l'élément po (litt. "plus»), et naj-2 «le plus» pour le superlatif. Il s'agit d'éléments autonomes, précédant le mot gradable, mais quand ils se combinent avec des adjectifs et des adverbes (c'est leur usage le plus fréquent), ils leur sont reliés par un trait d'union (po-bărz "plus rapide», naj-bărz «le plus rapide»).

Le bulgare est donc différent des langues romanes qui, pour différencier le comparatif du superlatif, se servent principalement des trois stratégies suivantes:

- Le superlatif peut être obtenu par l'association du morphème du comparatif à l'article défini (2); sans celui-ci, on a affaire au comparatif (1):

1) Ese coche es más rápido. (espagnol)

Cette voiture est plus rapide.

2) Ese coche es el más rápido. (espagnol)

Cette voiture est la plus rapide.

- quand l'usage de l'article défini n'est pas possible, la distinction peut être faite moyennant l'explicitation du complément dont le mot introductif n'est pas le même (Bosque \& Brucart (1991) cités par Rohena-Madrazo (2007:4) parlent de «comparative» (3) et «superlative codas» (4) en espagnol):

3) el niño más rápido (que todos nosotros)

le garçon plus rapide (que tous nous)

le garçon plus rapide que nous tous

4) el niño más rápido (de todos nosotros) le garçon plus rapide (de tous nous)

le garçon le plus rapide de nous tous (Rohena-Madrazo, 2007:4)

- enfin, l'enchâssement du "complexe» (morphème du comparatif + mot gradable) dans une relative définie est une autre stratégie pour obtenir le superlatif (6) (l'exemple (5) n'ayant que l'interprétation comparative malgré la définitude du SN sujet):
5) $L a$
ragazza
è più
arrabbiata (italien)
la fille
est plus
fâchée

2 Étymologiquement, naj est formé de la préposition na «sur» et de l'élément pronominal $i$ d'origine probablement déictique (cf. Bălgarski etimologičen rečnik [Dictionnaire étymologique du bulgare] de Gréorgiev \& Duridanov, 1995). 
6) Maria ’̀ [la ragazza che è più arrabbiata] (italien) Marie est la fille qui est plus fâchée

Marie est la fille la plus fâchée (Loccioni, 2018: 102-103)

En résumé, comme il a été bien établi dans la littérature, les langues romanes n'ont pas de morphème spécifique pour le superlatif. Elles se servent généralement du comparatif accompagné de l'article défini (français, roumain) ou mobilisent d'autres stratégies pour aboutir à l'unicité requise par le superlatif (italien, langues ibéroromanes). Comme en bulgare, il y a deux morphèmes distincts pour le comparatif et le superlatif, aucune des stratégies présentées ci-dessus n'est nécessaire en soi. Premièrement, l'article défini n'est pas obligatoire (8):

7) Tazi kola po- bărza. (cf. 1) cette voiture est COMP rapide Cette voiture est plus rapide.

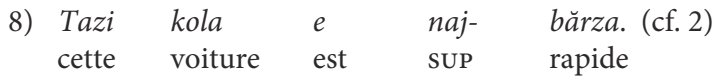
Cette voiture est la plus rapide.

Deuxièmement, comme la désambiguïsation se fait au niveau du morphème, la préposition qui introduit le comparant (pour le comparatif) (9) ou l'ensemble de comparaison (pour le superlatif) (10), est le plus souvent la même (il s'agit de ot, litt. "de»), à la différence de ce qu'on observe pour l'espagnol ((3) et (4) supra):
9) Tova dete e po- bărzo ot vsički nas. cet enfant est COMP rapide de tous nous Cet enfant est plus rapide que nous tous.

10) Tova dete e naj- bărzo ot vsički nas. ce enfant est sup rapide de tous nous Cet enfant est le plus rapide de nous tous.

Et enfin, l'enchâssement dans une relative définie (cf. (6)) n'est pas nécessaire: le superlatif peut très bien être exprimé «at the sentential level» («au niveau phrastique») selon les termes de Loccioni (2018), comme en (8). 


\section{2. Éléments combinables avec le morphème du superlatif}

Naturellement, les adjectifs (qualificatifs et gradables) en emploi épithétique (11a) ou attributif (11b) et les adverbes (12) sont les catégories prototypiques qui se combinent avec naj-, pour exprimer le superlatif:

11) a. Tova e naj- hubava-ta ${ }^{3}$ pesen. ceci est sup belle-la chanson C'est la plus belle chanson.

$\begin{array}{rllll}\text { b. Tazi pesen } & e & \text { naj- } & \text { hubava(-ta). } \\ \text { cette chanson } & \text { est } & \text { sup } & \text { belle(-la) }\end{array}$

Cette chanson est la plus belle.

12) Tja tiča naj- bărzo.

elle court sup vite

Elle court le plus vite.

Les verbes et les noms peuvent également recevoir le superlatif, comme dans beaucoup de langues. Tout comme le roumain $(13,14)$ et contrairement au français (cf. Van Peteghem, 2021), le bulgare (15, 16) se sert d'un quantifieur dans ces deux cas: mnogo «beaucoup", qui est l'équivalent du quantifieur roumain mult. De plus, à la différence $\mathrm{du}$ français, dans ces deux langues le nom «au superlatif» $(14,16)$ est introduit directement - le quantifieur se comportant comme un adjectif:

13) Maria lucrează cel mai mult. (roumain) Maria travaille $\mathrm{CEL}^{4}$ plus beaucoup Maria travaille le plus.

14) Ion a intervievat cel-e mai mult-e fete. (roumain) Ion a interviewé CEL-F.PL plus beaucoup-F.PL filles-F.PL Ion a interviewé le plus de filles. (Van Peteghem, 2021: 20)

3 L'article défini bulgare (qui est enclitique) se réalise à la manière d'une flexion variable en genre et en nombre qu'on ajoute directement à la fin d'un adjectif ou d'un nom (ici on doit écrire hubavata), mais pour la clarté de la présentation, nous le séparerons systématiquement par un tiret.

4 Pour les propriétés du «déterminant défini fort» $c e l$ en roumain, se référer à Van Peteghem (2021: 20-21) et aux références qu'elle donne, notamment (Teodorescu, 2007; Dobrovie-Sorin \& Giurgea, 2013). 
15) Marija raboti naj- mnogo.

Maria travaille sup beaucoup

Maria travaille le plus.

16) Ivan izpita naj- mnogo studenti.

Ivan interrogea sup beaucoup étudiants

Ivan a interrogé le plus d'étudiants.

Mais dans les deux cas illustrés par les exemples ci-dessus, il s'agit de superlatifs de quantité: celle d'une action ou d'un ensemble de référents. En revanche, Il existe, en bulgare la possibilité de faire précéder directement certains verbes et noms par naj, autrement dit de les «mettre au superlatif» sans l'intermédiaire d'un quelconque quantifieur:

17) Naj običa-m da hod-ja na more. sup aime-1sG que vais-1sG à mer J'aime le plus aller à la mer.

18) Toj e naj junak ot vsički măže. il est sup héros de tous hommes Il est le plus brave de tous les hommes.

Les noms et les verbes qui peuvent fonctionner ainsi comportent sémantiquement l'idée de propriété au sens large. De ce fait, Burov (1977) et Choroleeva (2007) les rapprochent des superlatifs de qualité.

Le superlatif peut aussi s'appliquer à des groupes prépositionnels de manière (19) ou à sens locatif (20), comme c'est le cas dans d'autres langues:

19) Čovek naj [s udovolstvie] razkazva za uspehi-te si. homme sup avec plaisir raconte pour réussites-les ses On raconte avec le plus de plaisir ses (propres) réussites.

20) Ivan hvărli stotinka-ta naj [v sreda-ta na fontan-a]. Ivan jeta centime-le sup dans milieu-le de fontaine-la Litt. Ivan a jeté le centime le plus au milieu de la fontaine.

\subsection{Les compléments du superlatif}

L'ensemble de comparaison, quand il est explicité (et directement dénoté), prend la forme d'un SP dont la tête est occupée par un petit paradigme de prépositions à sens partitif (comme dans les autres 
langues, cf. Van Peteghem, 2021): ot «de», (v)sred «parmi» et (iz) $m e \check{z} d u$ "d'entre», la première (ot) étant la plus neutre:

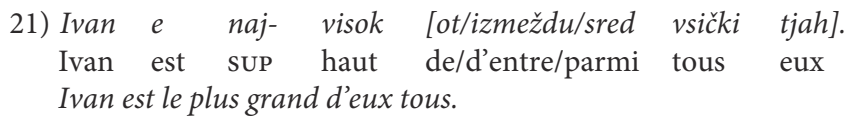

Sans surprise, des compléments locatifs ou temporels (sous la forme de SP, d'adverbes ou de subordonnées circonstancielles) peuvent également être mobilisés pour référer à l'ensemble de comparaison, en isolant soit une entité parmi d'autres dans un lieu donné (22), soit le degré le plus élevé d'une qualité chez un individu à un moment temporel donné (23):

22) Kazvat, če e naj- hubava-ta $v$ grad-a . disent-ils, que est sup belle-la dans ville-la Les gens disent qu'elle est la plus belle dans la ville. (Choroleeva, 2007: 162)

23) $T o j e$ naj- vesel, kogato e rabotil dobre prez Il est sup joyeux quand est travaillé bien pendant den-ja. journée-la Il est le plus joyeux quand il a bien travaillé pendant la journée.

Enfin, des subordonnées relatives (définies) peuvent également «restreindre» l'ensemble de comparaison (cf. Van Peteghem, 2007 pour le français):

24) Tja e naj-hubava-ta žena, kojato poznav-am. elle est sup belle-la femme que connais-1sG C'est la plus belle femme que je connaisse.

5 Ici grada «la ville» est dans un emploi métonymique (la ville pour ses habitants), mais la ville peut également être conçue simplement comme un lieu, auquel cas on aura affaire au plus haut degré d'une qualité chez un seul et même individu en fonction des moments où il se trouve dans le lieu en question. Dans ce cas le prédicat doit normalement être de type "stage level» (prédicat d'état, appelé aussi épisodique): Tja e naj-iznervena, kogato e v grada «Elle est le plus énervée quand elle est dans la ville» et le défini est proscrit (tout comme en (23)), ce qui correspond à l'usage du défini neutre en français (nous y reviendrons dans 3.4. infra). 


\section{Le superlatif en bulgare et l'article défini}

\subsection{Généralités}

Comme nous l'avons déjà dit dans l'introduction, en bulgare l'article défini n'est pas associé en soi à l'expression du superlatif. La première explication qui vient spontanément à l'esprit est qu'il y a un morphème spécifique pour le superlatif, différent de celui du comparatif: de ce fait, l'usage de l'article défini n'est pas nécessaire pour lever l'ambiguité entre les deux catégories. Cependant, cette explication se heurte au fait que dans une langue comme l'anglais, qui, tout comme le bulgare, possède des morphèmes distincts pour le comparatif et le superlatif, le défini est presque toujours associé à ce dernier, même avec les adverbes (25), les verbes (26) et les adjectifs en emploi prédicatif (27):

25) Anna runs the fastest.

26) Who works the most?

27) a. Anna was the fastest.

b. This film was the most interesting. (Larrey et Rivière, 2005: 226-232)

$\mathrm{Si}$, en (27a et b), la présence de the pourrait s'expliquer dans le cadre de l'hypothèse de Matushansky (2008) selon laquelle les adjectifs fast et interesting seraient en emploi épithétique (avec un $\mathrm{N}$ implicite reconstituable: respectivement girl et film, et on aboutirait à des DP), il n'en va pas ainsi de (25) et (26) où la structure ne prévoit aucun nom implicite (cf. Coppock \& Strand, 2017: 18, note 11, qui critiquent la généralisation de l'hypothèse de Matushansky en disant: "the impossibility of overt nouns in adverbials would appear to remain a problem, both for English and for French »). Si on regarde maintenant les équivalents bulgares des exemples anglais ci-dessus, on constate que l'article est proscrit avec les adverbes (28) et les verbes (29), alors qu'il est possible (mais pas obligatoire) avec les adjectifs en position attributive (30):

28) Ana tiča naj-bărzo(*-to). (cf. 25)

Ana court sup vite(-*le)

Ana court le plus vite.

29) Koj raboti naj-mnogo $\left({ }^{*}\right.$-to)? (cf. 26)

qui travaille sup beaucoup $\left({ }^{\star} l e\right)$ ?

Qui travaille le plus? 
30) a. Ana beše naj-bărza(-ta). (cf. 27)

Ana était sup rapide(-la)

Ana était la plus rapide.

b. Tozi film beše naj-interesen/interesni-ja.

ce film était sup intéressant(-le)

Ce film était le plus intéressant.

Il devient clair avec ces données que l'article défini en bulgare ne peut apparaître qu'avec des adjectifs, puisque c'est uniquement dans ce cas qu'une projection DP est envisageable. Larreya \& Rivière (2005) notent cependant que dans tous les exemples anglais ci-dessus (sauf 27b) l'article défini est facultatif ${ }^{\circ}$ : Anna runs fastest, Who works most? et Anna was fastest sont possibles et ont la même interprétation. En revanche, quand un $\mathrm{N}$ est ajouté dans le dernier cas, l'usage de l'article est de rigueur: Anna was ${ }^{*}$ (the) fastest girl (c'est la même chose en bulgare). L'optionalité de l'article montre que dans les cas où il n'est pas structurellement justifié (absence de DP), le morphème du superlatif peut suffire pour exprimer l'unicité. En fin de compte, le bulgare et l'anglais ne sont donc pas très différents, ce qui serait la conséquence logique du fait qu'ils ont des morphèmes spécifiques pour le comparatif et le superlatif (rappelons que pour les exemples examinés ci-dessus les langues romanes ou bien généralisent l'usage du défini, ou bien se servent d'autres stratégies pour exprimer la définitude).

Revenons à l'exemple (27b), où le défini est obligatoire, malgré la position prédicative de l'adjectif: en anglais la présence de l'article défini avec most est en effet nécessaire pour obtenir l'interprétation superlative d'un adjectif sans quoi on aboutit à la lecture élative (This film was most interesting "Ce film était très intéressant»). L'alternance présence/absence d'article est également de rigueur avec most dans le cas des superlatifs de quantité (avec des noms), pour désambiguïser entre lecture superlative (relative (31) et lecture proportionnelle (32):

31) The local library has got the most books on the subject.

La bibliothèque locale a le plus de livres sur le sujet.

32) The local library has got most books on the subject.

La bibliothèque locale a la plupart des livres sur le sujet.

6 Krasikova (2012: 408) fait la même remarque pour les superlatifs qu'elle identifie comme "prédicatifs» et «adverbiaux». 
En bulgare, où la lecture proportionnelle exprimée par le superlatif est marginale, le superlatif de quantité se construit sans déterminant, ce qui est conforme au fait relevé dans la littérature (Szabolcsi, 1986, entre autres) selon lequel les superlatifs de quantité n'ont que la lecture relative (ex. 16 que nous répétons ici pour faciliter la lecture): 16) Ivan izpita naj- mnogo studenti.
Ivan interrogea sup beaucoup étudiants
Ivan a interrogéle plus d'étudiants.

Reste à expliquer pourquoi en anglais l'article défini est possible avec les adverbes (25) et les verbes (26), alors qu'en bulgare il est totalement exclu dans ces cas. Pour rendre compte de ce phénomène, nous pouvons bien sûr suivre Krasikova (2012) qui distingue deux valeurs (ou statuts) du défini au sein des syntagmes superlatifs: le défini «canonique» qui occupe la tête d'un DP (et est toujours réalisé) et celui qui marque la définitude dans le cadre d'une projection DegP (Degree Phrase), sans forcément l'associer à une structure nominale (et qui peut ne pas être réalisé, selon les langues et les structures). En anglais, l'article défini avec les verbes et les adverbes aurait cette deuxième valeur, alors qu'en bulgare cette valeur serait associée à l'absence d'article. Néanmoins, la question reste de savoir pourquoi l'article de DegP (avec les verbes et les adverbes) peut être réalisé en anglais mais pas en bulgare. Nous pensons que le statut morphologique de l'article dans l'une et l'autre langue respectivement a son importance: rappelons qu'en bulgare l'article est sous la forme d'une flexion (marquant la définitude) qu'on ajoute au premier élément linéaire d'un SN pour le rendre défini (cf. Moskovsky, 2004; Mostrov, 2010)

33) a. kola-ta

voiture-la

la voiture

b. nova-ta kola

nouvelle-la voiture

la nouvelle voiture

c. goljama-ta nova kola

grande-la nouvelle voiture

la grande nouvelle voiture

Il est à noter cependant que si l'adjectif est modifié par un adverbe, même si celui-ci se trouve être le premier élément linéaire du SN entier, 
l'article enclitique ne se rattachera pas à lui mais bien à l'adjectif(qui est le premier élément linéaire pouvant recevoir la flexion du défini):

34) Prekaleno ADV čuvstvitelna-ta duša na Kalitko

Démesurément sensible-la âme de Kalitko

L'âme démesurément sensible de Kalitko

(exemple adapté de Hadžimarčev, Le pastoureau Kalitko, 1955)

Ces données confirment encore une fois que l'article défini enclitique en bulgare ne peut se combiner qu'avec des noms et des adjectifs. Cette contrainte semble découler du fait qu'il se réalise comme une flexion: une flexion modifie la forme d'un mot et le mot en question doit être strictement compatible avec la catégorie encodée dans la flexion. Or, un adverbe ne peut tout simplement pas avoir de forme fléchie. En revanche, en anglais, l'article défini est un morphème indépendant antéposé (de surcroît invariable), qui n'affecte en rien la forme des mots qui le suivent, et peut avoir par conséquent une combinatoire beaucoup moins contrainte, puisqu'il opère au niveau syntaxique. De ce fait, rien ne l'empêche d'être visible en tant que déterminant de DegP sans structure nominale (comme en français, mais pas en italien). Il est à noter qu'en roumain, l'article qui est toujours associé au superlatif est le morphème indépendant $\mathrm{cel}$ ( $\mathrm{cf}$. note 4) et non l'article défini enclitique (qui a les mêmes propriétés que celui du bulgare); le bulgare n'utilisant pas un article correspondant à cel dans le superlatif, il recourt au seul article défini enclitique, très contraint toutefois au niveau de la combinatoire, comme nous venons de le voir.

\subsection{La distinction entre position épithétique et attributive}

La distinction entre position épithétique et attributive est très discutée dans la littérature sur les superlatifs puisqu'elle est fortement corrélée à la question de l'apparition de l'article défini et/ou de son «statut», en fonction des langues. Ainsi, Loccioni (2018) soutient que, dans une langue comme l'italien, l'article défini apparaît uniquement quand l'adjectif est dans une position épithétique, que le nom soit explicite (35a) ou implicite (35b, 35c), alors qu'il est absent quand l'adjectif est dans une position prédicative $(36 \mathrm{a}, 36 \mathrm{~b})^{7}$. Par conséquent,

7 Notons qu'il n'y a pas de consensus dans la littérature sur la syntaxe des superlatifs postnominaux en (36a): selon Loccioni (2018: 105), il s'agirait de relatives 
on a affaire à un DP dans le premier cas (le morphème du superlatif à l'intérieur de ce DP entraînant la lecture absolue), alors que dans le second il n'y a pas de structure nominale, et le morphème du superlatif fait partie du prédicat:

\begin{tabular}{|c|c|c|c|c|c|c|c|}
\hline 5) a. Sale & $\grave{e}$ & il & più & grosso & gatto & bianco. & \\
\hline Sale & est & le & plus & gros & chat & blanc. & \\
\hline b. Sale & $\grave{e}$ & il & più & grosso. & & & \\
\hline Sale & est & le & plus & gros. & & & \\
\hline c. Sale & $e$ & il & pì̀ & grosso & & gatt & hi. \\
\hline Sale & est & le & plus & gros & de.les & chats & blancs. \\
\hline
\end{tabular}
36) a. il gatto bianco più grosso le chat blanc plus gros le chat blanc le plus gros
b. lapersona che mi rende più felice la personne qui me rend plus heureux la personne qui me rend le plus heureux (Loccioni, 2018: 6)

En français en revanche, l'article défini est présent même en position prédicative, comme on peut le voir dans les traductions françaises de (36a et b). Dans cette langue donc, que le déterminant soit celui d'un DP ou d'un DegP, il est toujours réalisé.

Le bulgare se comporte grosso modo comme l'italien: l'article défini est présent quand on a une projection nominale (37), alors qu'il est absent en position prédicative $(38)^{8}$ :
37) Ivan $e \quad n a j$-sposobni-jat
Ivan est sup capable-le ouvrier
Ivan est l'ouvrier le plus capable.

prédicatives réduites, analyse qui est en adéquation avec l'absence d'article observée tout au moins en italien et en espagnol, alors que pour Kayne (2008), Matushansky (2008) et Alexiadou (2014), il s'agirait de structures nominales à déterminant réalisé (français) ou non réalisé (italien, espagnol).

8 La structure en (36a) n'a pas d'équivalent en bulgare, raison pour laquelle nous ne l'exemplifions pas. En bulgare il est possible par contre d'utiliser, à la place du syntagme superlatif postnominal, une relative à attribut sans déterminant (bjala-ta kotka, kojato e naj-debela «le chat blanc qui est le plus gros»), ce qui irait dans le sens de Loccioni (2018), pour qui les superlatifs postnominaux sont de nature prédicative (cf. note 7). 
38) Ivan Focus me pravi naj-štastliv. (cf. 36b)

Ivan me rend supheureux

Ivan me rend le plus heureux.

Par contre, quand il n'y a pas de nom réalisé et que la projection nominale occupe une position prédicative (35b, 35c), le bulgare se distingue de l'italien puisque l'article est le plus souvent absent, et la structure reste purement prédicative:

39) Ivan e naj-sposoben. (cf. 35b)

Ivan est sup capable

Ivan est le plus capable.

40) Ivan e naj-sposoben ot rabotnici-te. (cf. 35c)

Ivan est sup capable de ouvriers-les

Ivan est le plus capable des ouvriers.

En italien, la contrainte de l'apparition de l'article défini (qui entraîne une projection nominale) s'explique ici par l'absence de morphème spécifique pour le superlatif (sans le défini on aboutirait au comparatif). Même la présence d'un complément dénotant l'ensemble de comparaison, comme en (35c), peut ne pas être suffisante pour lever l'ambiguité entre les deux catégories, puisque les compléments du comparatif et du superlatif peuvent être introduits par la même préposition di (sans le défini, (35c) se traduirait par Sale est plus gros que les chats blancs, et dans ce cas Sale ne ferait pas partie des «chats blancs»). En bulgare, comme le morphème du superlatif est distinct de celui du comparatif, le déterminant n'est pas nécessaire, mais sa présence reste tout de même possible, du moins lorsqu'on a affaire à la lecture absolue (au sens de Szabolcsi (1986) et Heim (1999)), ce qui est le cas de (39) 9 et de (40). Une structure nominale et une structure prédicative peuvent donc alterner en bulgare, du moins quand la position du syntagme superlatif dans son entier est prédicative (cf. 3.4. infra).

Dans ce qui suit nous examinons les différentes structures superlatives en bulgare et les conditions de leur association avec l'article

9 En (39), la lecture absolue est favorisée par le fait qu'on a affaire à un prédicat individuel (permanent, caractérisant). En effet, il est difficile d'imaginer que le degré de la capacité d'Ivan puisse varier en fonction de différentes circonstances qui joueraient le rôle de focus, dans le cadre de lectures relatives (??Ivan e naj-sposoben, kogato raboti sam «Ivan est le plus capable quand il travaille seul»). 
défini en fonction des configurations morphosyntaxiques où elles apparaissent, de leur position syntaxique (épithétique ou attributive) et de leur interprétation (lecture absolue, relative ou élative). Nous nous focalisons sur les structures où un adjectif est présent, puisqu'elles sont les seules où la question de la présence/absence du défini est pertinente.

\subsection{Les superlatifs épithétiques}

Ces superlatifs, qui se présentent sous la formule $n a j+A d j+N$, peuvent apparaître, en fonction du contexte et de l'interprétation, avec l'article défini (41) ou sans lui (42):

41) Ivana ima [naj-goljama-ta kăšta], kojato săm viždal. Ivana a sup grande-la maison que suis-1sg vu Ivana a la plus grande maison que j'aie jamais vue.

42) Ivana ima [naj-goljama kăšta] sred pop zvezdi-te. Ivana a supgrande maison parmi pop stars-les Ivana a la plus grande maison parmi les vedettes de variété.

Disons d'emblée que le contraste entre (41) et (42) est dans la lecture associée au superlatif: absolue en (41) et relative (ou comparative) en (42). Dans le premier exemple, avec le défini (et la relative), l'ensemble de comparaison est fourni par le NP kashta «maison», et le superlatif a une portée à l'intérieur du DP (parmi toutes les maisons que j'ai vues, la maison d'Ivana est la plus grande). En revanche, dans le second, sans le défini, l'ensemble de comparaison (= les vedettes de variété) est à l'extérieur du NP, le superlatif ayant une portée qui dépasse le cadre de celui-ci, le focus étant le sujet Ivana ${ }^{10}$. Notons cependant que l'apparition du défini est possible en (42), sans changement d'interprétation, mais on sait que dans beaucoup de langues (dont l'anglais) les superlatifs relatifs peuvent se construire avec le défini, lequel correspond néanmoins (en forme logique) à l'indéfini (cf. Szabolcsi, 1986). Le bulgare peut donc, pour ce qui est des superlatifs relatifs, garder en structure de surface l'indéfini de la forme logique, mais il peut aussi, ce qui est la règle dans d'autres langues, lui substituer le défini (qui serait celui de DegP, selon Krasikova, 2012), du moins dans certains cas (cf. 3.3.3. infra).

10 À la manière de Szabolcsi (1986), on pourrait paraphraser (42) par la structure comparative Ivana a une maison plus grande que celles des autres vedettes de variété. 
Il est évident que la présence ou l'absence de l'article est corrélée aux lectures associées aux superlatifs, la tendance qui semble se dégager du contraste entre (41) et (42) étant que les superlatifs définis favoriseraient la lecture absolue, alors que les indéfinis favoriseraient la lecture relative. Pour vérifier/affiner cette tendance, nous examinerons le comportement des superlatifs dans des configurations syntaxiques

(i) qui exigent des $\mathrm{SN}$ définis,

(ii) qui exigent des $\mathrm{SN}$ indéfinis,

(iii) qui sont compatibles aussi bien avec des $\mathrm{SN}$ définis qu'indéfinis.

\subsubsection{Configurations syntaxiques où l'article défini est obligatoire}

Remarquons que, dans des structures qui exigent la présence de SN arguments obligatoirement définis, l'article défini sera également de rigueur dans le cas de $\mathrm{SN}$ comportant le morphème du superlatif. Il en est par exemple ainsi dans les cas suivants (nous nous sommes inspiré de Tilkov et al., 1983):

- Le SN est sujet dans une structure prédicative à attribut qualifiant:

43) $\operatorname{Kniga}^{*}(-t a)$ e/izgležda interesna. livre ${ }^{\star}(-\mathrm{le}) \quad$ est/semble intéressant Le livre est/semble intéressant.

44) Naj-debela $^{*}(-$ ta $) \quad k n i g a \quad e \quad$ interesna. sup épais ${ }^{*}(-$ le) livre est intéressant Le livre le plus épais est intéressant.

- Le SN est COD d'un verbe à préfixe perfectivant, qui présente le processus comme achevé avec présupposition d'une quantité donnée d'un ensemble (limité) de référents:

45) Ivan šte do-bere kruši*(-te).

Ivan FUT PERF-cueillir poires ${ }^{\star}($ les $)$

Ivan cueillera ( $=$ finira de cueillir) les poires.

46) Ivan šte do-bere naj-uzreli*(-te) kruši.

Ivan FUT PERF-cueillir sUP mûres ${ }^{*}(-$ les $)$ poires

Ivan cueillera (= finira de cueillir) les poires les plus mûres.

- Le SN est argument interne de avoir impersonnel et est doublé par la forme réduite du pronom personnel correspondant (à l'accusatif):

47) $\operatorname{Deca}^{*}(-t a)$ gi ima na snimka-ta.

enfants ${ }^{\star}(-\mathrm{les})$ les ily a sur photo-la

Les enfants sont sur la photo ou Les enfants, ils y sont sur la photo. 
48) $\mathrm{Naj}_{-}$visoki*(-te) deca gi ima na snimka-ta. sup grands*(-les) enfants les ilya sur photo-la Les plus grands enfants sont sur la photo.

- Le SN est un complément partitif (introduit par ot «de»), qui présuppose l'existence d'un ensemble dont on prélève une portion:

49) Ivan si vze ot $h l j a b^{*}(-a)$.

Ivan se.DAT prit de pain*(-le)

Ivan s'est servi du pain. (du: article défini contracté et non partitif indéfini)

$\begin{array}{rlllll}\text { 50) Ivan } & \text { si } & \text { vze } & \text { ot } & \text { naj-topli }{ }^{*}(-j a) & \text { hljab. } \\ \text { Ivan } & \text { se.DAT } & \text { prit } & \text { de } & \text { sup chaud }{ }^{*}(-\mathrm{le}) & \text { pain }\end{array}$

Ivan s'est servi du pain le plus chaud.

Il semble bien que dans tous ces exemples, même dans ceux où un focus (extérieur donc au DP superlatif) est virtuellement disponible ((46) et (50) Ivan, (48) snimkata "la photo»), la lecture absolue est la seule possible. Ceci découle du fait que l'article défini, puisqu'il est exigé par la structure, est sans ambigüité celui d'un $\mathrm{DP}^{11}$, et ne peut donc pas correspondre à celui d'un DegP (ou indéfini en forme logique, selon Szabolcsi, 1986). En effet, les superlatifs ci-dessus (nous prenons comme exemple (46)) ne peuvent ni se construire avec un complément qui «sélectionne» un focus (46'a), ni se paraphraser par des comparatifs à article indéfini $\left(46^{\prime} \mathrm{b}\right)$ :

46') a. ??Ot vsički, Ivan Focus šte dobere naj-uzreli-te kruši

De tous, Ivan FUT PERF-cueillir sup mûres-les poires

b. ${ }^{\star}$ Ivan šte dobere po- uzreli kruši ot drugi-te

Ivan FUT PERF-cueillir COMP mûres poires que autres-les

studenti

étudiants

\subsubsection{Configurations syntaxiques où l'article défini est proscrit}

Les configurations syntaxiques où l'article défini est proscrit correspondent à des cas pour lesquels on a affaire à des SN qui occupent des positions non argumentales, comme par exemple dans

11 Et, en tant que tel, il opère un «test d'unicité» au niveau du DP (cf. Loccioni, 2018: 63), lequel se comporte comme un «îlot» (degree island selon Tomaszewicz, 2013: 230 , note 3 ) où le morphème du superlatif est «piégé». 
les constructions existentielles, dans des locutions à verbe support, ou encore quand le $\mathrm{SN}$ fait partie de groupes prépositionnels à valeurs circonstancielles variées. Dans tous ces cas, si on a affaire à des SN superlatifs, ils resteront indéfinis:

- Le SN est complément de imam «avoir» en emploi impersonnel («il y a $)$ :

51) Dnes

$$
v \quad \text { severna Bălgaria šte ima obilni }
$$

aujourd'hui dans septentrionale Bulgarie FUT ily a abondantes snegovaleži.

chutes-de-neige

Aujourd'hui dans la Bulgarie septentrionale il y aura des chutes de neige abondantes.

52) Dnes v severna Bălgaria šte ima najaujourd'hui dans septentrionale Bulgarie FUT ilya sUP obilni snegovaleži. abondantes chutes-de-neige Aujourd'hui dans la Bulgarie septentrionale il y aura les chutes de neige les plus abondantes (ou il neigera le plus).

- Le N fait partie d'une expression impersonnelle (collocationnelle) à verbe imam «avoir» (ima nadežda «il y a de l'espoir», ima smisăl «il y a du sens», etc.):

53) Tuk ima nadežda da namer-iš săkrovište-to. ici ilya espoir que trouves-2sG trésor-le Ici il y a de l'espoir/des chances que tu trouves le trésor.

54) Tuk ima naj-goljama nadežda da namer-iš săkrovište-to. ici ilya sup grand espoir que trouves-2sg trésor-le Ici il y a le plus grand espoir que tu trouves le trésor.

- Le $\mathrm{N}$ fait partie d'une construction personnelle à verbe support (le plus souvent imam "avoir»), comme imam vina "être coupable/ responsable», imam predstava «avoir une idée, connaître», etc. :

55) Socialisti-te imat vina za finansova-ta kriza $v$ socialistes-les ont faute pour financière-la crise dans strana-ta. pays-le

Les socialistes sont responsables de la crise financière dans le pays.

56) Socialisti-te imat naj-goljama vina za finansova-ta kriza socialistes-les ont sup grande faute pour financière-la crise $v$ strana-ta. 
dans pays-le

Les socialistes ont la plus grande responsabilité dans la crise financière dans le pays.

D'ailleurs, l'expression take a picture discutée par Szabolcsi (1986: 245) est parallèle à ce type de structures en bulgare. Szabolcsi note que le $\mathrm{N}$ picture est indéfini dans cette expression (57a et l'impossible 57b) et que même si l'article défini est utilisé quand on a affaire à un syntagme superlatif $(57 \mathrm{c})$, il n'en reste pas moins indéfini (comme valeur, en forme logique), ce qui est en conformité avec la lecture relative (avec focus sur who) obtenue en (57c):

57) a. Who did you take a picture of?

b. ${ }^{\star}$ Who did you take the/every picture of?

c. Who ${ }_{\text {Focus }}$ did you take the best picture of?

En bulgare, l'équivalent pravja snimka (litt. «faire photo», c'est-à-dire "prendre une photo») se comporte comme les expressions énumérées ci-dessus (cf. notamment celle en (56)). Même quand le "complément" snimka 'photo' intègre un syntagme superlatif (58), le défini n'est pas approprié, et tout comme en (57c), le focus est sur kogo «qui»:

58) $\mathrm{Na}$ kogo Focus naprav-i naj-hubava snimka?

à qui fis-2sg sup belle photo?

De qui as-tu pris la plus belle photo?

- Enfin, le N reste indéfini dans un SP complément circonstanciel de manière:

59) Život-ăt im minava [v nesgodi]. vie-la leur.DET.Poss passe dans difficultés Leur vie est rencontre des difficultés. (Tilkov et al., 1983: 140)

60) Život-ăt im minava [v naj-golemi nesgodi]. vie-la leur.DET.Poss passe dans sup grandes difficultés Leur vie est rencontre les plus grandes difficultés.

Nous pouvons d'emblée noter que la lecture absolue n'est disponible dans aucun des exemples bulgares supra à SN superlatifs non définis. Sauf en (60) que l'on discutera infra, on a affaire à des lectures relatives, le focus pouvant être un CC de temps (dnes «aujourd'hui» en (52)), de lieu (v severna Bălgaria «dans la Bulgarie septentrionale» en (52), tuk «ici» en (54)), un sujet (Socialistite "Les socialistes» en (56)) ou un COI (na kogo «à qui» en (58)). Ceci n'a rien d'étonnant, vu que pour obtenir la lecture absolue (dans les superlatifs épithétiques), il 
faut un «vrai» DP, avec un article défini qui restreint la portée du morphème du superlatif au seul DP, comme nous l'avons vu supra. Or, en l'absence d'un tel DP, seules des lectures relatives peuvent être disponibles ${ }^{12}$, comme en témoigne l'exemple bulgare (61):
61) Ivan Focus kupi [DPnaj-skăpa torta] za MeriFocus. Ivan acheta sup chère tarte pour Meri Ivan a acheté la tarte la plus chère pour Meri. (Tomaszewicz, 2013:230)

Cette phrase peut signifier soit qu'Ivan (le focus) a acheté une tarte plus chère que celles qu'ont achetées (pour Meri) d'autres garçons, soit qu'Ivan a acheté une tarte plus chère pour Meri (le focus) que celles qu'il a achetées pour d'autres filles, mais jamais qu'Ivan a acheté pour Meri la tarte la plus chère parmi un ensemble prédéfini de tartes comparées relativement à leur prix. Citons Tomaszewicz (2013: 230) pour plus de precision: "When the definite is absent [...] relative readings obtain, with suitable prosody, whereas the absolute reading is unavailable».

Revenons à l'exemple (60). Il n'illustre ni la lecture relative, ni l'absolue, puisqu'il n'y a ni ensemble de comparaison, ni focus. Tout semble indiquer qu'on a simplement affaire à une lecture élative puisque naj pourrait être remplacé (sans véritable changement de sens) par des adverbes d'intensité forte comme izključitelno "excessivement, particulièrement» (60'a), mais il peut également être omis, auquel cas on ne perdrait que le degré d'intensité (60’b):
60') a. Život-ăt im
minava vie-la leur.DET.Poss passe dans
izključitelno golemi nesgodi.
difficultés
Leur vie rencontre des difficultés particulièrement grandes.
b. Život-ăt im minava $v$ golemi nesgodi vie-la leur.DET.Poss passe dans grandes difficultés Leur vie rencontre de grandes difficultés.

En revanche, la suppression de naj ou son remplacement par des adverbes d'intensité dans les exemples (52, 54, 56 et 58) entraîneraient

12 On aura affaire à des sentential scope superlatives " constructions superlatives qui ont une portée sur toute la phrase» et à des definite effect contexts «contextes à effet de définitude» où un focus est obligatoire, cf. Szabolcsi (1986). 
un changement de sens notable, puisqu'on perdrait la lecture superlative (relative) au profit de l'élatif.

\subsubsection{Configurations syntaxiques compatibles avec des SN définis aussi bien qu'indéfinis}

Il est bien connu que les compléments ou les sujets de beaucoup de verbes peuvent être définis ou indéfinis, en fonction de la visée référentielle de leurs arguments (existence posée ou présupposée). On peut acheter du/le/ce pain, escalader une/la/cette montagne, posséder une/la/cette maison (compléments), de même qu'un/le/ce garçon peut venir ou dire quelque chose (sujet). Nous commencerons par examiner les cas de compléments pour ensuite discuter de ceux où le $\mathrm{SN}$ est sujet et nous dirons enfin quelques mots sur les $\mathrm{SN}$ en position prédicative.

- Le SN est en position d'objet:

Revenons à l'exemple (61) supra de Tomaszewicz (2013) où le SN superlatif ne comporte pas d'article défini. Contrairement aux autres exemples de la section précédente (3.3.2), le SN complément direct de (61) peut également apparaître avec le défini, comme l'a bien noté cette auteure:

61') Ivan Focus kupi [DPnaj-skăpa-ta torta] za Meri ${ }_{\text {Focus }}$. Ivan acheta sup chère-la tarte pour Meri Ivan a acheté la tarte la plus chère pour Meri.

L'alternance entre article défini et absence d'article peut s'expliquer précisément par le fait qu'un verbe comme acheter accepte des COD aussi bien définis qu'indéfinis. Au niveau de l'interprétation, (61') diffère de (61) en ceci que la lecture absolue devient disponible: Ivan a acheté pour Meri la tarte la plus chère parmi un ensemble prédéterminé de tartes. Ceci est en conformité avec le statut du défini en tant que déterminant de DP qui isole un individu à partir d'un ensemble d'individus de même type, sur la base du plus haut degré d'une propriété partagée par les individus de l'ensemble en question. Mais (61') garde aussi les deux lectures relatives disponibles en (61), avec respectivement un focus sur Ivan et sur Meri. La présence de l'article défini ne bloque donc pas les lectures relatives au profit de la seule lecture absolue, phénomène que nous avons déjà observé dans la discussion de l'exemple (42) supra. Mais pour qu'en bulgare des lectures relatives (externes donc au DP) soient disponibles en 
présence du défini, tout semble indiquer qu'il faut que la configuration syntaxique soit compatible, en elle-même, avec des SN indéfinis ${ }^{13}$. Par conséquent, il n'y a pas d'obstacle à ce que le défini, dans le cas des lectures relatives, s'analyse comme un indéfini en forme logique - conformément à l'hypothèse proposée par Szabolcsi (1986) - et qui correspond au défini qui marque la définitude de l'argument de degré (DegP) selon Krasikova (2012). Le défini de (61') est donc ambigu entre son statut de base (déterminant de DP) et celui d'un déterminant «de degré», compatible avec l'indéfinitude sémantique du SN. En fonction de son interprétation, on a respectivement affaire à la lecture absolue ou à des lectures relatives. Le célèbre exemple ambigu John climbed the highest mountain, traduit en bulgare, révèle le même phénomène, avec les deux lectures disponibles (i) et (ii):

62) Ivan izkači naj-visoka-ta Ivan escalada sup haute-la montagne

Ivan a escaladé la plus haute montagne.

(i) Ivan a escaladé la plus haute montagne du monde (lecture absolue)

(ii) Ivan a escaladé une montagne plus haute que celles qu'ont escaladées les autres alpinistes (lecture relative)

Toutefois, à la différence de l'anglais, le SN superlatif COD en bulgare peut se construire sans l'article défini, auquel cas seule la lecture relative est disponible, avec un focus sur Ivan (ii). Ceci est prouvé par le fait qu'en l'absence du défini, nous pouvons uniquement ajouter un complément dénotant l'ensemble de comparaison dont Ivan fait partie (63a), mais pas un complément, tel que na sveta "dans le monde entier», qui impose la lecture absolue (63b):

63) a. Ot vsički učastnici, Ivan Focus izkači naj- visoka planina de tous participants Ivan gravit sup haute montagne De tous les participants, Ivan a gravi la plus haute montagne.

$\begin{array}{rllll}\text { b. }{ }^{\star} \text { Ivan } & \text { izkači } & \text { naj-visoka planina na svet-a } \\ \text { Ivan } & \text { gravit } & \text { sup haute } & \text { montagne } & \text { dans }\end{array}$

13 Nous avons déjà vu en 3.3.1. que seule la lecture absolue est disponible quand la configuration syntaxique exige exclusivement des $\mathrm{SN}$ définis. 
- Le SN est en position de sujet:

Prenons comme exemple le sujet du verbe montrer qui peut être aussi bien indéfini (64) que défini (65):

64) Edin rabotnik mi pokaza kak raboti mašina-ta. Un ouvrier me.DAT montra comment fonctionne machine-la Un ouvrier m'a montré comment fonctionne la machine.

65) Rabotnik-ăt mi pokaza kak raboti mašina-ta. Ouvrier-le me.DAT montra comment fonctionne machine-la L'ouvrier m'a montré comment fonctionne la machine.

Pourtant, si le sujet est un SN superlatif, l'indéfini est proscrit (66a) ${ }^{14}$, seul le défini étant possible (66b):

66) a. ${ }^{\star}$ Edin naj- sposoben rabotnik mi pokaza kak

Un sup capable ouvrier me.DAT montra comment raboti mašina-ta

fonctionne machine-la

b. Naj- sposobni-jat rabotnik mi pokaza kak sup capable-le ouvrier me.DAT montra comment raboti mašina-ta.

fonctionne machine-la

L'ouvrier le plus capable m'a montré comment fonctionne la machine.

L'impossibilité d'un SN superlatif indéfini sujet semble contredire la généralisation esquissée ci-dessus concernant les SN objet. Mais nous ne pensons pas qu'il s'agisse d'un contre-exemple, puisque de toute évidence seule la lecture absolue peut être associée à la position de sujet: en effet, même si on ajoute un adverbial qui, dans d'autres circonstances, pourrait jouer le rôle de focus, il n'affecterait en rien l'interprétation d'un SN superlatif sujet:

67) Včera naj- sposobni-jat rabotnik mi pokaza kak hier sup capable-le ouvrier me.dat montra comment raboti mašina-ta. fonctionne machine-la Hier l'ouvrier le plus capable m'a montré comment fonctionne la machine.

14 L'astérisque de (66a) correspond à la non-disponibilité de lectures superlatives, mais notons que la phrase pourrait à la rigueur être acceptée si on interprétait najsposoben comme exprimant l'élatif («un ouvrier très capable»). 
L'adverbial včera «hier» ne fait que situer dans le temps l'événement dont il est question, sans interférer d'une quelconque manière avec le superlatif. La présence obligatoire du défini s'explique alors par la lecture absolue qu'impose la position de sujet (cf. l'analyse des superlatifs épithétiques de Loccioni, 2018 pour l'italien). En effet, seul un complément dénotant l'ensemble de comparaison dont fait partie le sujet (comme ot celija zavod «de toute l'usine» en (68)) peut être ajouté en lien avec l'interprétation du superlatif:

68) Naj-sposobni-jat rabotnik ot celi-ja zavod mi pokaza sup capable-le ouvrier de toute-la usine me.DAT montra

kak raboti mašina-ta.

comment fonctionne machine-la

L'ouvrier le plus capable de toute l'usine m'a montré comment fonctionne la machine.

La situation des $\mathrm{SN}$ sujets rappelle celle des $\mathrm{SN}$ toujours définis discutés dans 3.3.1., à ceci près qu'ici c'est la lecture exigée qui impose le défini et non la configuration syntaxique elle-même.

- Le SN est en position d'attribut:

Ce cas rappelle le précédent, puisqu'un $\mathrm{SN}$ indéfini peut très bien occuper la position attributive (69), mais dès qu'on a affaire à un SN superlatif, le défini apparaît (70):

69) Ivan e sposoben rabotnik.

Ivan est capable ouvrier

Ivan est un ouvrier capable.

70) Ivan e naj-sposobni-jat rabotnik.

Ivan est sup capable-le ouvrier

Ivan est l'ouvrier le plus capable.

Il est parfois possible d'omettre le défini, comme dans l'exemple littéraire ci-dessous, mais, tout comme pour la position de sujet, on aboutira à la lecture élative:

71) Bojčo, az te znaja dobre, ti si naj-blagoroden čovek. Boicho je te connais bien tu es sup noble homme Boicho, je te connais bien, tu es un homme noble au plus haut degré.

(I. Vazov, Sous le joug, 1890)

La contrainte du défini en (70) s'explique de nouveau par la lecture absolue du syntagme (cf. (35a) supra de Loccioni, 2018), mais aussi par le fait que le SN superlatif ne dénote pas simplement une propriété du 
sujet ou l'appartenance de celui-ci à une classe de référents (comme en (69) et (71)), mais bien la propriété unique qui caractérise le sujet, en instaurant un rapport d'identité référentielle entre les deux constituants (cf. Riegel, 1985).

L'identité référentielle et la lecture absolue semblent aller de pair, puisque le référent du sujet, se confondant avec celui du SN attribut, ne peut qu'être inclus dans l'ensemble de comparaison que dénote le $\mathrm{N}$ du syntagme superlatif.

Dans le paragraphe suivant nous poursuivrons l'analyse des superlatifs en position prédicative, en examinant ensemble les $\mathrm{SN}$ à nom explicite ou implicite et les AP (Adjectival Phrases, Syntagmes Adjectivaux) afin de révéler certains contrastes significatifs.

\subsection{Les superlatifs nominaux et adjectivaux en position prédicative}

Nous avons déjà vu (cf. 3.2.) qu'en bulgare, à la différence de l'italien, une projection nominale n'est pas nécessairement présente dans ce que Loccioni (2018: 114) appelle «[the] main clause superlatives»(superlatifs figurant dans des propositions indépendantes). En effet, l'adjectif peut rester sans déterminant (ce qui est d'ailleurs plus fréquent), qu'on soit ou non en présence d'un complément dénotant l'ensemble de comparaison:

72) Ivan e naj-sposoben/sposobni-jat (ot vsički rabotnici).

Ivan est sup capable/capable-le (de tous ouvriers)

Ivan est le plus capable (de tous les ouvriers).

En position prédicative, une structure nominale à nom implicite et une structure AP peuvent donc alterner en bulgare, sans changement d'interprétation (à ceci près qu'avec le défini l'expression de l'unicité est peut-être renforcée). En revanche, dès qu'on explicite le nom, l'usage du défini est de rigueur, comme nous l'avons vu supra (70). Notons néanmoins que l'alternance défini/absence de défini n'est possible que quand on a affaire à la lecture absolue. Il est vrai que dans la littérature on associe souvent la lecture absolue à la présence d'un DP puisque c'est celui-ci qui fournit l'ensemble de comparaison, mais Krasikova (2012: 408) n'exclut pas cette lecture pour ce qui est des "main clause superlatives» (comme dans son exemple (13b) John is (the) tallest), donc sans structure nominale obligatoire, en disant que dans ces cas «alternatives in comparison classes are always shaped after 
the subject». De même, Loccioni (2018), qui privilégie la corrélation entre superlatifs prédicatifs et lectures relatives, analyse les superlatifs postnominaux (du type la fille la plus belle) comme prédicatifs, alors qu'ils n'ont que la lecture absolue. Cette auteure (communication personnelle) attribue également le même type d'interprétation au superlatif prédicatif de l'exemple (6) supra qu'on répète ici:

6) Maria ̀̀ [la ragazza che è più arrabbiata]. (italien) Marie est la fille qui est plus fâchée

Marie est la fille la plus fâchée. (Loccioni, 2018: 102)

Mais l'alternance défini/absence de défini n'est pas toujours possible en position prédicative. Le défini est proscrit en bulgare quand on a affaire à des lectures relatives où le focus est réalisé sous la forme de différents circonstants (de temps et de lieu le plus souvent), le référent du sujet n'étant pas interprété comme faisant partie d'un ensemble de référents de même type que lui ((23) supra, répété ci-dessous). Comme nous l'avons déjà dit (notes 5 et 9 ), ce type de lecture s'obtient le plus souvent avec des prédicats d'état (stage level), puisque ce qui est comparé, ce sont différents degrés d'une propriété attribuée au même individu, à des moments ou dans des lieux différents:

23) Toj e naj-vesel, kogato e rabotil dobre prez den-ja. il est sup joyeux quand est travaillé bien pendant journée-la Il est le plus joyeux quand il a bien travaillé pendant la journée.

En (23), le syntagme superlatif ne peut être qu'adjectival: il est impossible d'y ajouter un nom (à la différence de (72), avec le défini), ce qui correspond à l'impossibilité, en anglais, de faire suivre l'adjectif par l'élément pronominal one dans ce type de lecture, où le défini est de surcroît optionnel:

73) Mary was (the) prettiest ( ${ }^{*}$ one) yesterday $_{\text {Focus. }}$

$=$ Mary was prettier yesterday than on any other relevant day. (Loccioni, 2018: 71)

En français, il est à noter que dans ce type d'interprétation le défini se réalise sous sa forme "neutre ${ }^{15}$ (cas de discordance de genre, «gender mismatch»), fait relevé dans les grammaires (Riegel, Pellat \&

15 Situation qui rappelle celle du néerlandais, cf. Van Peteghem \& Lagae, dans ce volume. 
Rioul, 2009: 625; Grevisse, 1986: \$950) et illustré par Van Peteghem (2021: 21):

74) C'est avec son premier mari qu'elle était le plus heureuse.

(Van Peteghem, 2021: 21, son exemple 42b')

Pour rendre compte de l'invariabilité du déterminant, Riegel, Pellat \& Rioul (2009) analysent [le+plus] comme une locution adverbiale, alors que le défini conserverait «son statut d'article» $(=\mathrm{DP})$ dans les cas où il s'accorde (elle était la plus heureuse à la fête) et qui instancient la lecture absolue.

En italien (et en espagnol), tout comme en bulgare, pour obtenir la lecture relative l'article défini ne peut pas être utilisé (75), car s'il est utilisé, on obtiendra automatiquement la lecture absolue (76). La présence/absence d'article permet donc de lever l'ambiguité entre les deux lectures:

75) L'anno Focus in cui Maria fu più felice l'année en laquelle Maria était plus heureuse

L'année où Marie était LE plus heureuse

(= plus heureuse que n'importe quelle autre année, lecture relative)

76) L'anno in cui Maria fu la più felice l'année en laquelle Maria était la plus heureuse

L'année où Marie était LA plus heureuse

(= la plus heureuse d'un ensemble de femmes, lecture absolue)

(Loccioni, 2018: 116)

Pour récapituler, en suivant Krasikova (2012), on peut rendre compte des contrastes observés ci-dessus de la façon suivante (cf. aussi 3.1. supra): que ce soit en français, en italien, en anglais ou en bulgare, l'article défini, dans la lecture absolue est celui d'un DP (l'adjectif étant épithète) et donne forcément lieu à une projection nominale, que le nom soit explicite ou implicite. Dans ce cas, il est toujours présent et s'accorde (dans les langues où il est variable) avec le sujet et/ou le nom du DP. Loccioni (2018: 117) donne de ce type de DP superlatifs la structure suivante:

$$
\left[{ }_{D P} \mathrm{D}\left[\left[{ }_{A P} \ldots\right] \mathrm{N}\right]\right]
$$

En revanche, dans les lectures relatives (23), (73), (74), (75) et (76), l'article, qui exprime la définitude au sein d'une projection DegP dont il est la tête, n'est pas (forcément) associé à un DP, et peut par conséquent se combiner avec des constituants purement prédicatifs 
(adjectivaux mais aussi adverbiaux, comme dans Il court le plus vite), ce qui est illustré par la formule suivante (Loccioni, 2018: 117):

$$
\text { [AP [DegP D -est degree ] A ] ] }
$$

$\mathrm{Au}$ niveau de sa réalisation matérielle, l'article de DegP peut être présent ou absent selon les langues et les structures. En bulgare et en italien il est absent (dans les structures prédicatives); en français et en néerlandais il apparaît sous sa forme neutre, tandis qu'en anglais il est facultatif. La variante de (72) sans le déterminant est intéressante puisqu'elle montre, comme il a été noté supra, que la lecture absolue peut être obtenue malgré l'absence de projection nominale.

\section{Conclusion}

Cette étude a montré que l'usage de l'article défini en bulgare n'est pas directement lié à l'expression du superlatif ${ }^{16}$, lequel est encodé par le morphème spécifique naj, différent de celui du comparatif ( $p o$ ). Nous avons montré que l'article défini, qui se présente sous la forme d'une flexion, ne peut être employé qu'en présence d'une projection nominale, que le nom soit réalisé ou implicite: il est, par conséquent, impossible avec les verbes et les adverbes. De ce point de vue, la situation en bulgare est compatible avec l'hypothèse de Matushansky (2008), ou tout du moins avec le postulat selon lequel la présence de l'article défini implique obligatoirement la présence d'une structure nominale.

Pour déterminer les conditions d'apparition ou d'absence de l'article défini en lien avec les différentes lectures disponibles, nous nous sommes focalisé sur des constructions où un adjectif était présent, car c'est uniquement dans ces cas que la question de la présence du déterminant se pose en bulgare. Nous avons ainsi examiné des structures où l'adjectif occupe une position épithétique, le SN entier pouvant être dans une position argumentale ou prédicative, et d'autres où l'adjectif reste purement prédicatif.

L'examen de ces différentes configurations a révélé que quand on est en présence de SN superlatifs sans article défini, que le contexte

16 La présence/absence du défini dépend en grande partie de la configuration morphosyntaxique, ce qui rappelle la situation en arabe syrien (Hallman, 2021 ce volume). 
exige ou non l'indéfinitude, seules des lectures superlatives relatives sont disponibles (avec présence obligatoire de focus), à côté de la lecture élative. La portée du morphème du superlatif dépasse dans ces cas le seul cadre du SN: on a affaire à des sentential scope superlatives (constructions superlatives qui ont une portée sur toute la phrase). En revanche, les SN superlatifs définis peuvent instancier aussi bien la lecture absolue que des lectures relatives, en fonction du statut du déterminant: quand celui-ci est la tête d'un DP, il marque l'unicité à l'intérieur de ce DP et on aboutit à la lecture absolue, alors que quand il peut alterner avec l'indéfini (qui, en bulgare, n'est le plus souvent pas réalisé), il aurait le statut du défini associé à l'expression d'un degré et occuperait la tête d'un DegP (Krasikova, 2012). Des cas d'ambiguités peuvent alors se présenter, ce qui arrive dans des configurations syntaxiques compatibles aussi bien avec des $\mathrm{SN}$ définis qu'indéfinis (cf. 3.3.3.); en revanche, dans des structures qui exigent en elles-mêmes des $\mathrm{SN}$ définis (cf. 3.3.1.), le déterminant associé au superlatif ne peut être que celui d'un DP (à lecture absolue donc).

Quant aux superlatifs prédicatifs, deux cas de figure se présentent: on a d'un côté des AP qui peuvent alterner avec des DP à nom implicite pouvant être explicité, et d'un autre côté des AP qui ne peuvent jamais alterner avec une structure nominale. Dans le premier cas on a affaire à la lecture absolue, alors que dans le second ce qui est isolé est le plus haut degré d'une propriété d'un seul et même individu, en fonction de différentes circonstances qui jouent le rôle de focus (lecture relative). Le premier cas montre d'ailleurs que la lecture absolue peut être associée à un AP superlatif dans une position prédicative, même si la tendance générale qui émerge de la littérature est l'association de cette lecture à la présence d'une structure nominale.

Pour résumer, l'article défini enclitique bulgare peut bien avoir, dans le superlatif, les deux statuts identifiés par Krasikova (2012), à savoir celui de la tête d'un DP et celui de la tête d'un DegP, mais dans les deux cas, pour qu'il puisse être réalisé, une structure nominale doit être présente. Nous avons vu que sans structure nominale, l'apparition de l'article est impossible en bulgare, au point de se demander si on doit forcément stipuler la présence d'un quelconque article (implicite) dans les emplois autres que nominaux. En effet, comme le dit Matushansky (2008: 62, note 36), l'unicité/la «maximalité» exigée par le superlatif peut être encodée par le seul morphème superlatif, surtout quand 
celui-ci est différent du morphème du comparatif, ce qui est le cas du bulgare.

Naturellement, nous n'avons pas examiné tous les cas de superlatifs en bulgare, laissant pour un travail ultérieur les superlatifs de quantité, les superlatifs modaux et la frontière entre l'expression du superlatif et celle de l'élatif.

\section{Bibliographie}

ABNEY P. (1987), The English Noun Phrase in its sentential aspect, PhD dissertation, MIT, Cambridge, Massachusetts.

ALEXIADOU A. (2014), Multiple determiners and the structure of DPs, Amsterdam/Philadelphia, John Benjamins.

BOSQUE I. \& BRUCART J.M.(1991), QP Raising in Spanish superlatives, unpublished draft, Madrid, Universidad Complutense de Madrid.

BUROV S. (1977), Kăm văprosa za stepenuvaneto na imena i glagoli v bălgarskija ezik [Sur la question des degrés des noms et des verbes en langue bulgare], Rocznik Slawistyczny 38:1, 57-69.

BUROV S. (1981), Gramatičeskata kategorija "stepenuvane" $v$ săvremennija bălgarski knižoven ezik (funkcionalen analiz) [La catégorie grammaticale "degré" dans la langue littéraire bulgare moderne], thèse de doctorat, Université de Veliko Tărnovo.

BUROV S. (1982), Kăm văprosa za stepenuvaneto na prilagatelnite imena i narechiata $\mathrm{v}$ săvremennija bălgarski knižoven ezik [Sur la question des degrés des adjectifs et des adverbes dans la langue littéraire bulgare moderne], Bălgarski ezik 32:2, 104-110.

CHOROLEEVA M. (2007), Semantičnata kategorija "stepen" i nejnoto izrazjavane $v$ bălgarskija ezik [La catégorie sémantique "degré" et son expression dans la langue bulgare], Sofia, Prof. Marin Drinov.

COPPOCK E. \& STRAND L. (2017), Most vs. the most in languages where the more means most, Boston University Libraries, Open $B U,<$ https://hdl.handle.net/2144/27852>.

GEORGIEV V.\& DURIDANOV I. (eds) (1995), Bălgarski etimologichen rečnik [Dictionnaire étymologique du bulgare], vol. 4, Sofia, Prof. Marin Drinov.

GREVISSE M. (1986), Le Bon Usage, 12e édition, Paris, Duculot. 
DOBROVIE-SORIN C. \& GIURGEA I. (eds.) (2013), A reference grammar of Romanian: Volume 1: The noun phrase, Amsterdam/ Philadelphia, John Benjamins.

HEIM I. (1999), Notes on superlatives, Unpublished draft, Cambridge, MIT.

HALLMAN P. (2021), Conditions on Definiteness in Arabic Superlatives, ce volume Scolia 35, 69-92.

KLEIBER G. (1992), Article défini, unicité et pertinence, Revue romane 27:1, 61-89.

KRASIKOVA S. (2012), Definiteness in superlatives, in Aloni M., Kimmelman V., Roelofsen F., Sassoon G.W., Schulz K. \& Westera M. (eds.), Logic, Language and Meaning, Berlin, Heidelberg, Springer, 411-420.

KAYNE, R. (2008), Some preliminary comparative remarks on French and Italian definite articles, in Freidin R., Otero C.P. \& Maria Luisa Zubizarreta M.L. (eds.), Foundational Issues in Linguistic Theory, Cambridge, MIT Press, 291-321.

LARREYA P. \& RIVIERE C. (2005), Grammaire explicative de l'anglais, $3^{\mathrm{e}}$ édition, Paris, Pearson Education France.

LOCCIONI N. (2018), Getting "the most" out of Romance, PhD dissertation, University of California at Los Angeles.

MATUSHANSKY O. (2008), On the attributive nature of superlatives, Syntax 11: 1, 26-90.

MOSKOVSKY C. (2004), Optional movement of Bulgarian possessive clitics to I: Some implications for Binding Theory, in Mišeska Tomic O. (ed.), Balkan Syntax and Semantics, Amsterdam/ Philadelphia, John Benjamins, 221-233.

MOSTROV V. (2010), Inalienable possession in French and in Bulgarian, in Lahousse K., Lamiroy B. \& Van Goethem K. (eds.), French syntax in contrast, Linguisticae Investigationes 33:2, 239252.

PANCHEVA R. \& TOMASZEWICZ B.(2012), Cross-linguistic Differences in Superlative Movement out of Nominal Phrases, in Arnett N. \& Bennett R. (eds.), Proceedings of the 30th West Coast Conference on Formal Linguistics, Somerville, MA, Cascadilla Proceedings Project, 292-302.

PAŠOV P. (2015), Bălgarska Gramatika [Grammaire du bulgare], Plovdiv, Presses Hermes.

RIEGEL M. (1985), L'adjectif attribut, Paris, PUF.

RIEGEL M., PELLAT J.-C., RIOUL, R. (2009), Grammaire méthodique $d u$ français, 4 e édition, Paris, PUF. 
ROHENA-MADRAZO M. (2007), Superlative movement in Puerto Rican Spanish and General Spanish, NYU Working Papers in Linguistics 1, 1-32.

SZABOLCSI A. (1986), Comparative superlatives, MIT Working Papers in Linguistics 8, 245-265.

TEODORESCU V. A. (2007), Attributive superlatives in Romanian, in Alboiu G., Avram A., Avram L. \& Isac D. (eds.), Pitar Moş: A building with a view. Papers in honor of Alexandra Cornilescu, Bucharest, Editura Universitatii din Bucuresti, 249-264.

TILKOV D. et al. (1983), Gramatika na săvremennija bălgarski knižoven ezik: morfologija, tom 2 [Grammaire de la langue littéraire bulgare moderne, vol. 2], Sofia, Presses de l'Académie bulgare des sciences.

TOMASZEWICZ B. (2013), Focus association in superlatives and the semantics of -est, in Aloni M., Franke M. \& Roelofsen F. (eds.), Proceedings of the 19th Amsterdam Colloquium, 226-233.

VAN PETEGHEM M. (2007), Les relatives et les adjectifs à valeur superlative, in Charolles M., Fournier N., Fuchs C. \& Lefeuvre F. (éds), Parcours de la phrase: Mélanges offerts à Pierre Le Goffic, Paris, Ophrys, 99-104.

VAN PETEGHEM M. (2021), Comparatives and superlatives in the Romance languages, in Loporcaro M., Gardani F., Cabredo Hofherr P.,ClaesJ.,Dufter A., Maiden M. \&RainerF.(eds.), Oxford Research Encyclopedia of Linguistics, Oxford University Press, $<$ https://doi.org/10.1093/acrefore/9780199384655.013.668>.

VAN PETEGHEM M. \& LAGAE V. (2021), Attributs adjectivaux superlatifs en français et en néerlandais et l'accord de l'article, Scolia 35, 39-68. 